\title{
La Liberte de la Presse dans le Contexte Electoral au Sud- Kivu
}

\author{
Par Bahati Cibambo Aline*
}

\section{INTRODUCTION}

Dans beaucoup de pays africains, à l'instar de la RDC, les élections libres sont un nouveau phénomène. ${ }^{1}$ Le rôle que les médias sont appelés à jouer pendant la période électorale dans un Etat qui se veut de droit est indéniable. Les candidats doivent pouvoir faire connaître leurs projets politiques. Dans cette optique, les médias ont la capacité de pouvoir atteindre le plus grand nombre d'électeurs en un temps record. Cela veut dire que les médias ${ }^{2}$ devaient être utilisés comme plateforme permettant aux partis politiques et aux candidats d'interagir avec le public. Ce qui doit conduire à la prise de conscience par les électeurs de la valeur de leur vote afin de faire un choix éclairé dans les urnes. Pour jouer pleinement ce rôle, les médias doivent exercer librement, recueillir des nouvelles et donner de l'espace pour que ceux qui le souhaitent puissent exprimer leurs opinions sur les questions des élections. $^{3}$

Quarante-sixans après l'accession de la RDC à l'indépendance, ${ }^{4}$ cette dernière $a$, après près de trois décennies sans élections, organisé, en 2006, les élections présidentielles, législatives pluralistes. Cinq ans plus tard, c'est-à-dire en 2011, le pays avait renouvelé l'expérience. A cette occasion, la population a pu, à nouveau, élire le Président de la République et les députés nationaux, mais cette fois pas ceux provinciaux. Ce fut dans le cadre de la mise en œuvre de la démocratie voulant que le peuple se choisisse lui-même ses dirigeants. ${ }^{5}$ Pour celles censées être organisées en 2016, l'année vient de s'écouler sans qu'elles ne

* Assistante à 1’Université Catholique de Bukavu, e-mail : bahati.cibambo@ucbukavu.ac.cd

1 Vincent DARRACQ et Victor MAGNANI, « Jeux de pouvoir en Afrique : le Nigéria et l'Afrique du Sud face à la crise ivoirienne », Politique étrangère, Vol 76, n² 2 , juin 2006.

2 Le mot «médias » est compris ici dans le sens de la presse écrite,orale et audiovisuelle.C'estl'ensemble des supports d'information et de communication qui atteignent facilement toute une société. Bref, il s'agit de tout support de diffusion de l'information (radio, télévision, presse imprimée, livre, ordinateur, vidéogramme, satellite de communication, etc.)Parler de la presse tout court, englobe aussi celle orale et écrite.

3 Reporters sans Frontières, Guide pratique du Journaliste en période électorale, Organisation internationale de la Francophonie, 2009, p.14.

4 La République Démocratique du Congo autre fois Congo-Belge puisqu'elle fut à l'époque une colonie de la Belgique entre 1908-1960. Voir à ce sujetLissia JEURISSEN, Histoire coloniale et nomadisme heuristique : « Du Congo de papa » au «Bled», Bruxelles, 2006,p. 34.

5 Florent GUENARD, « La promotion de la démocratie : une impasse théorique? », Esprit n³41, janvier 2008, p. 122. 
l'aient été et des incertitudes planent au tour de la date de leur tenue éventuelle. ${ }^{6}$ Et dans ces incertitudes, le climat politique est tendu où la majorité, l'opposition dispersée et une frange de la société civile après avoir trouvé un consensus après un énième dialogue sur une transition à la fin du mandat du président actuel se font une guerre pour le partage des postes. $^{7}$

Le fonctionnement de la démocratie repose sur une libre expression des opinions par les citoyens. Cette liberté d'expression se matérialise notamment par la liberté de la presse, qui permet d'informer les citoyens et de leur donner les moyens de comprendre les enjeux de la société à l'exemple de ceux électoraux. ${ }^{8}$

L'on peut ainsi s'interroger sur la question de savoir quel est le rôle joué par la presse au Sud-Kivu dans la période électorale et si la liberté de la presse consacrée par les instruments juridiques tant nationaux qu'internationaux est effective dans la province considérée.

La liberté de la presse étant une liberté fondamentale, ${ }^{9}$ elle est protégée tant par un nombre important d'instruments juridiques internationaux et régionaux des droits humains auxquels la RDC a adhéré que par l'arsenal juridique congolais. Indépendamment de ce fait, le constat global de l'exercice de cette liberté surtout en période électorale est que ce pays est caractérisé par un climat électoral tendu amenuisant ainsi l'exercice de la liberté de la presse. Au Sud-Kivu, particulièrement, ce droit serait de plus en plus remis en question et son exercice effectif, un grand défi pour la RDC qui se veut un Etat de droit. Durant les deux premières élections de 2006 et 2011, et dans la fièvre des incertitudes sur la tenue effective de celles de 2016, des cas de musellement de la presse sous diverses formes sont de plus en plus nombreux.

L'analyse part du point de vue temporel part de 2006, année de l'entrée en vigueur de la Constitution de la RDC et de la tenue des élections pluralistes depuis plusieurs décennies. Elle va jusqu'à 2016, année au cours de laquelle les prochaines élections étaient censées être organisées.

Pour ce faire, nous présenterons respectivement l'état des lieux de l'exercice de la liberté de la presse au Sud-Kivu (A), en abordant aussi la question des limites à son exercice et

6 En principe, le scrutin présidentiel sensé être au convoqué au 19 septembre ne l'a plus été et aucun calendrier définitif n'a été publié par la Commission électorale nationale et indépendante. Le consensus trouvé au dialogue parl'accord signé le 19 octobre 2016 parle du maintien au pouvoir du président actuel et de l'organisation des élections en avril 2018. Cet accord a été revu par un autre signé le 31 décembre sous l'égide de la CENCO et prévoit queles élections soient organisées fin décembre 2017.

7 Indépendamment du consensus trouvé à ce dernier dialogue, l'accord n'a pas encoretrouvé l'inclusivité souhaitéepuisque la classe politique est toujours déchirée. Autre fois c'était surla question de l'après 19 décembre 2016, date à laquelle le président actuel était sensé cédé la place à un autre,élu démocratiquement et aujourd'hui sur le partage des postes.

8 Electoral Knowledge network, "Médias et élections", www.oceproject.org, accédé dernièrement le 07 septembre 2016.

9 Préambule du Code d'éthique et de déontologie du journaliste, Journal officiel, $47^{\text {ème }}$ numéro spécial du 20 juin 2006. 
les conditions dans lesquelles les médias jouent ce rôle. Ce qui nous conduira à présenter le tableau des violations de la liberté de la presse constatées au cours de ces dix dernières années. Nous parlerons ensuite du rôle des médias en période électorale et des perspectives pour une presse plus libre au Sud-Kivu (B).

\section{A. ETAT DES LIEUX DE L'EXERCICE DE LA LIBERTE DE LA PRESSE AU SUD-KIVU EN PERIODE ELECTORALE}

L'analyse de l'état des lieux de la liberté de la presse au Sud-Kivu nous amène tout d'abord à examiner la portée de ce principe et ensuite ses limites avant de voir la manière dont cette liberté s'exerce en pratique. Ce qui nous permettra de nous faire une idée sur les conditions dans lesquelles la presse exerce sa mission surtout en période électorale par rapport au rôle attendu d'elle.

\section{Portée et limites de la liberté de la presse}

1. Portée du principe

Comme indiqué plus haut, la liberté de la presse, en tant que droit fondamental sous lequel se fonde tout Etat de droit, est consacrée par nombreux textes juridiques internationaux auxquels la RDC a adhéré. Tel est le cas de la Charte africaine des droits de l'homme et des peuples (CADHP), du Pacte International relatif aux Droits Civils et Politiques (PIDCP) ou encore de la Déclaration Universelle des Droits de l'Homme (DUDH). Il est également consacré en droit interne par la Constitution telle que modifiée par la loi $n^{\circ} 11 / 002 \mathrm{du}$ 20/01/2011 portant modification de certains articles de la Constitution du 18 février $2006^{10}$ et d'autres textes de lois comme la loi n 96-002 du 22 juin 1996 fixant les modalités d'exercice de la liberté de la presse. ${ }^{11}$ Pour le Sud-Kivu d'une manière particulière, i lest consacré par l'édit portant protection des journalistes et défenseurs des droits de l'homme. ${ }^{12}$

La liberté de la presse est l'un des principes fondamentaux des systèmes démocratiques qui repose sur la liberté d'expression et la liberté d'opinion. A cet effet, l'art. 19 du Pacte international relatif aux droits civils et politiques dispose :

«Nul ne peut être inquiété pour ses opinions. Toute personne a droit à la liberté d'expression; ce droit comprend la liberté de chercher, de recevoir et de répandre des in-

10 Art 24 de la Constitution modifiée par la loi $n^{\circ} 11 / 002$ du20/01/2011 portant modification de certains articles de la Constitution du 18février 2006, Journal officiel, $52^{\text {ème }}$ numéro spécial de 2011.

11 Art. 8de la loi n96-002 du 22 juin 1996 fixant les modalités d'exercice de la liberté de la presse, Journal officiel, numéro spécial d'Août 2001.

12 Art 4 et suivant de l'Edit provincial portant protection des défenseurs des droits humains et des journalistes, voy, www.protectioninternational.org/fr/2016/02/21/ accédé dernièrement le 20 août2016. 
formations et des idées de toute espèce, sans considération des frontières, sous une forme orale, écrite, imprimée ou artistique, ou par tout autre moyen de son choix $»^{13}$.

Cela implique que tout citoyen peut parler, écrire, imprimer librement. Ce droit s'exerce en tout temps y compris en période électorale. S'il est mal compris ou encore mal exercé, il peut être à l'origine des tensions et des troubles qui, à leur tour, pourraient porter atteinte à l'exercice d'autres droits. Les limites à l'exercice de la liberté de la presse ne portent pas sur une période au cours de laquelle elle ne peut être exercée mais sur la nécessité de prévenir des abus qui pourraient en découler. La liberté de la presse doit donc être exercée en tout temps, y compris pendant la période électorale. ${ }^{14}$

\section{Limites}

Qu'il s'agisse du PIDCP ou encore de la Constitution congolaise du 18 février 2006, les textes se rapportant à la liberté de la presse, ne se limitent pas seulement à en fixer le principe. Ils en donnent aussi les restrictions, car comme on le verra, le droit à la liberté de la presse n'est pas absolu. En effet, la liberté et la responsabilité de la presse vont de pair. ${ }^{15}$

Les limites à la liberté de la presse sont soit déterminées par la loi, au respect de laquelle veillent les tribunaux; soit justifiées par le sens des responsabilités dont tout professionnel, quel qu'il soit, doit inlassablement et immanquablement faire preuve. ${ }^{16}$ Tout en consacrant le droit à l'information à la population et garantissant en son article 24 la liberté de la presse, la Constitution de la RDC la tempère par la nécessité de préserver le respect des droits d'autrui, la sécurité nationale, l'ordre public, la santé publique et les bonnes mœurs. Elle fait également obligation aux médias dépendant de l'Etat de faire preuve d'objectivité et d'impartialité et de représenter de façon équitable la pluralité d'opinions. ${ }^{17} \mathrm{Ces}$ mêmes restrictions sont contenues aux articles. 19 du Pacte International relatif aux Droits Civils et Politiques et de la Déclaration Universelle des Droits de l'Homme (DUDH). En dépit de ces limites, la liberté de la presse demeure la règle et les restrictions, l'exception. ${ }^{18}$

Pour ce faire, le Conseil supérieur de l'audiovisuel et de la communication (CSAC), tout en garantissant la liberté de la presse, de l'information et de tout autre moyen de communication des masses, assure la protection de la presse, veille aussi au respect de la déon-

13 Art. 19 du Pacte international relatif aux droits civils et politiques du 16 décembre 1966, ce traité est entré en vigueur le 23 mars 1976, ratifié par la RD Congo le $1^{\text {er }}$ novembre 1967, journal officiel de la RDC, numéro spécial du 05 décembre 2002.

14 Reporters Sans Frontière (RSF), note 3, p. 15.

15 MichaelFLESHMAN, RDC: les médias défendent la démocratie, p. 9 consulté le 28 juin 2016.

16 Art 4 du Code de déontologie et d'éthique du journaliste, Journal officiel, $47^{\text {ème }}$ numéro du 20 juin 2006.

17 Art 24 de la Constitution modifiée par la loi $n^{\circ} 11 / 002$ du20/01/2011 portant modification de certains articles de la Constitution du 18 février 2006, Journal officiel, $52^{\text {ème }}$ numéro spécial de 2011.

18 Art 9de la loi n 96-002 du 22 juin 1996 fixant les modalités d'exercice de la liberté de la presse, Journal officiel, numéro spécial d'Août 2001. 
tologie en matière d'information, à l'accès équitable des partis politiques, des associations et de toute autre personne aux moyens officiels d'information et de communication. ${ }^{19}$

Les limites à la liberté de la presse ont pour but d'éviter des abus dans son utilisation, de manière que les médias ne véhiculent pas de fausses nouvelles ou des rumeurs infondées qui nuiraient à la réputation d'un individu, ou encore qu'ils ne propagent des messages de haine ou d'intolérance.

"Sont donc interdits à travers les médias, l'injure, la diffamation, la médisance, la calomnie, les accusations sans preuves, l'altérationdes documents, la déformation des faits, le mensonge, l'incitation à la xénophobie, à la haine (religieuse, ethnique, tribale, régionale ou raciale) ainsi que l'apologie de toute valeur négative, dans la pratique quotidienne de leur métier » 20

Ainsi, dans le contexte électoral, les médias qui s'adonneraient à pareille pratique, pour ou contre un candidat ou un parti politique, s'exposent à des sanctions et ne peuvent se justifier par la liberté dont ils jouissent dans cette profession, car être libre de s'exprimer ne signifie pas que l'on peut dire tout et n'importe quoi. ${ }^{21}$

Pour restreindre l'exercice de la liberté de la presse, les autorités doivent appliquer une législation officiellement connue. Ainsi, pour être pointé comme n'ayant pas respecté les limites de la liberté de la presse, les journalistes devraient savoir que tel comportement dépasse le champ de l'exercice de cette liberté. Cela s'explique par le fait que, les gouvernants ne peuvent limiter cette liberté comme cela peut bon leur sembler. ${ }^{22}$ Même lorsque ce sont eux qui sont visés par la critique, une gouvernance démocratique voudrait qu'ils ne puissent pas pour autant limiter cette liberté tout simplement pour se mettre à l'abri.

Les personnels des médias à leur tour, sont tenus au respect des normes régissant leur profession. Ils sont appelés à faire preuve d'un niveau élevé de professionnalisme, d'exactitude et d'impartialité dans leur couverture des élections. « La presse responsable ne doit pas être diffamatoire, dérivative, malveillante ou encore corrompue». ${ }^{23}$

Comme il sera démontré dans les lignes suivantes, le climat est souvent tendu à l'approche, pendant et même parfois après les élections et à cette occasion, nombreuses violations de la liberté de la presse sont décriées. Ce qui nous amène à analyser les conditions dans lesquelles cette liberté est exercée dans ce contexte particulier de la vie politique du Sud-Kivu.

19 Art. 8 de la loi organique ${ }^{\circ} 11 / 001$ du 10 janvier 2011 portant, attribution et fonctionnement du Conseil Supérieur de l'Audiovisuel et de la Communication, www.leganet.cd, dernièrement consulté le 10 Août 2016.

20 Art 5 Code d'éthique et de déontologie du journaliste congolais, journal officiel, numéro spécial du 20 juin 2006.

21 Reporteurs Sans Frontières, op cit, p. 27.

22 AiméKAYEMBE. et alii, Situation des médias en RDC, Paris, 2004, p. 27.

23 DanielCORNU, Journalisme et vérité pour une éthique de l'information, Genève, 1994, p. 38. 


\section{II. conditions d'exercice de la liberté de la presse au Sud-Kivu pendant les élections}

La liberté d'expression est un droit fondamental de l'homme. Elle a comme corolaire la liberté de la presse. ${ }^{24}$ Cependant, dans le paysage médiatique du Sud-Kivu, le fait d'avoir plusieurs textes consacrant la liberté de la presse ne suffit pas à en garantir l'effectivité. Sur le plan pratique, son application ne suit toujours pas. Des cas des violations de la liberté de la presse répertoriés au Sud-Kivu, sont de plus en plus nombreux. Ils vont des assassinats, des menaces, des intimidations, des fermetures, des entraves à la couverture médiatique des scrutins et campagnes, des censures, des pressions, des saisies des matériels de reportage, des agressions contre les journalistes, des refus d'accréditation, des arrestations pour intimider les personnes critiques à l'encontre du gouvernement sur les médias, aux blocages des messageries courtes (SMS) et internet, les pressions fiscales et financière... ${ }^{25}$ pour empêcher à certains partis de trouver de l'espace pour entrer en contact avec leur électorat.

Différents aspects de la liberté de la presse sont en jeu lorsqu'il s'agit d'élections. Pour cette période cruciale où le peuple se choisit ses dirigeants, dans un Etat de droit, cela implique notamment la protection contre la censure, contre les attaques arbitraires et l'ingérence, le libre accès à l'information nécessaire, le pluralisme des voix dans les médias, etc. ${ }^{26}$

Le journalisme consiste à trouver l'information, la traiter, la mettre en forme et la diffuser. Cela suppose un travail intellectuel pour vérifier la véracité d'une information et pour apprécier son intérêt pour le public. Ce qui demande, un plus haut niveau d'indépendance, de probité, d'équilibre, d'honnêteté et de neutralité. Mettre ces règles en pratique n'est toutefois pas simple. Les journalistes qui ne respectent pas ces règles s'exposent à des sanctions judiciaires. L'autonomie des médias suppose pour son effectivité, des moyens suffisants pour contourner les pièges et les risques auxquels les journalistes peuvent être confrontés. Sans ces moyens, les rédactions deviennent dépendantes de ceux qui désirent voir passer leur message pour partir en reportage, obtenir des images, réaliser des interviews. ${ }^{27}$ L'espace médiatique étant géré par plusieurs acteurs, dont le ministère de l'information et de la communication, les instances étatiques ou de corporation des médias en charge de la gestion et de régulation de la communication, des abus proviennent parfois même de ces mêmes acteurs. On constate nombreux dérapages des médias et de graves violations des règles de la déontologie de la profession de journalistes. La diffusion de l'infor-

24 UNESCO, Contribuer à la paix et au développement humain à l'ère de la mondialisation par l'éducation, les sciences, la culture et la communication, Paris, 2001, p. 43.

25 Réseau pour la réforme du secteur de sécurité et de justice (RRSSJ),JED récence 64cas de violation de la liberté de la presse depuis novembre 2011,www.rrssj.org, consulté le 24 octobre2016.

26 J. KHNEL, Enjeux de la bonne gouvernance et rôle des médias en RDC, Médias et bonne gouvernance, Kinshasa, 2005 p. 12.

27 Association des journalistes professionnels (APJ), les journalistes et leurs sources. Guide de bonnes pratiques, mars 2012, p.9. 
mation non vérifiée ou même fabriquée, fausse, mensongère, propagation des rumeurs, est courante en période électorale. ${ }^{28}$

Nombreux facteurs entrent en ligne de compte dans l'examen de l'exercice effectif de la liberté de la presse. C'est notamment le statut même des médias (1) qui, selon qu'ils sont publics ou privés a des incidences sur leur manière de traiter les informations et les messages à passer en période électorale. C'est également,le cas des entraves à la liberté de la presse au Sud-Kivu (b) parmi lesquelles les assassinats et agressions des journalistes (a), les emprisonnements (b), les menaces et intimidations (c), les fermetures et suspensions des radios (d) etc. régulièrement dénoncés dans la pratique.

\section{Le statut des médias au Sud-Kivu}

En règle générale, les médias qui assurent la couverture de la campagne, qu'ils soient de l'Etat ou privés devraient faire preuve d'impartialité dans leur présentation même pendant la période électorale. Cependant, persiste encore des personnalités soit privées soit publiques, qui continuent à interférer dans le travail des journalistes. ${ }^{29}$

En ce qui concerne les médias privés, ceux-ci regroupent les radios communautaires, associatives, académiques et scolaires, confessionnelles dites encore évangéliques. On se retrouve souvent en face des médias dont le financement dépend des capitaux privés qui peuvent vouloir modifier l'information en fonction de leurs intérêts. ${ }^{30}$ La question du financement des médias privés peut dans une large mesure altérer leur indépendance puisque très peu, si pas aucun média au Sud-Kivu n'est en mesure de se garantir un mode autonome de financement. $^{31}$

Parmi eux, il y en a qui ne naissent que dans le contexte particulier des propagandes et des campagnes électorales. Ils mettent alors en place des programmes leur accordant tout le temps voulu pour parler d'eux-mêmes et de leurs partis, choisissent à la loupe ceux de leur obédience qui peuvent comme eux accéder à leurs chaines. Dans ces conditions, on peut alors se retrouver en face des certains médias, surtout la radio et la télévision qui n'émettent que pendant la période de campagne et de propagande électorale. La poursuite de leurs activités après les élections est tributaire de l'issu du vote de ceux pour qui ils faisaient la pro-

28 Reporteurs Sans Frontières, note 3.26.

29 Document de la fédération internationale des journalistes : http://www.ifj.org, rubrique « quality in journalism », accédé dernièrementle 12/avril/2016.

30 Marie Soleil FRERE, " Pas d'élections démocratiques sans médias libres et pluralistes », Afrique contemporaine, avril 2011, p. 154.

31 Médias indépendants : entendu ici ceux qui n'ont aucune dépendance financière, politique ou religieuse.Lorsqu'ils sont publics, reçoivent le financements du gouvernement, s' ils sont privés ils sont tributaires des financements reçus de leurs propriétaires qui en même temps peuvent donner des directives allant dans le sens de soutenir tel candidat ou tel parti ou de restreindre l'espace à telle formation politique. 
pagande. Ainsi selon qu'ils gagnent ou qu'ils perdent, ses médias peuvent continuer ou arrêter leurs activités.

Ainsi, nombreux doivent suivre les directives données par les propriétaires ou encore les bailleurs selon le cas. Ils vont parfois à l'encontre des règles de l'éthique et de la déontologie qui gouvernent les professionnels des médias. C'est souvent les cas des médias privés conventionnels qui en même temps peuvent être des médias de parti s'ils appartiennent à un parti ou à un dirigeant ou un membre d'un parti afin de communiquer avec leurs membres, leurs sympathisants et le grand public. ${ }^{32}$ Idéalement, ils devraient se conformer aux normes ou règlements en vigueur indépendamment de leurs propriétaires.

$\mathrm{Au}$ sein de ses médias, la pratique consiste pour la plupart à ne pas payer le $\mathrm{fisc}^{33}$. Ce dernier ne leur demande de payer qu'à partir du moment où ils font des critiques acerbes contre le pouvoir en place ou contre telle autorité ou encore lorsqu'en période de campagne électorale, ils donnent la place à aux personnes défavorables au gouvernement pour s'exprimer. Ils reçoivent alors des notes de perception et menaces de fermeture ou de suspension pour non-paiement d'impôt. ${ }^{34}$ Une telle pratique, est un frein à la liberté de la presse rendant les médias vulnérables. Dans ces conditions, ils sont soumis à la crainte de s'exposer et se voir parfois légitimement contraint à se mettre en ordre avec le fisc. Ce qui les pousse à une autocensure.

Quant aux médias publics, financés par les deniers publics, ils devraient se conformer aux mêmes normes que tout autre média. La loi prévoit que, « les médias de l'Etat doivent fonctionner dans l'indépendance, la neutralité et le respect du principe de l'égalité de tous devant la loi. Ils ne peuvent en aucune circonstance compromettre l'exactitude et l'objectivité de l'information. La communication audiovisuelle publique est pluraliste. Elle ne peut en aucun cas, être monopolisée au profit d'une seule opinion ou d'un groupe d'individus $\gg .{ }^{35}$ En pratique cela veut dire qu'ils ne peuvent pas faire campagne pour tel ou tel autre parti. Constituant une ressource destinée à tous les électeurs, ils ne doivent pas prendre position en faveur de quelque parti politique que ce soit dans leur ligne éditoriale. Ils ne devraient pas se transformer en organe de propagande pour un parti politique donné mais devraient plutôt favoriser l'expression de la diversité d'opinions. « Ils ont en outre l'obligation de fournir une éducation civique et d'offrir une tribune aux différents partis $»{ }^{36}$

32 Reporters sans frontières, « liberté de la presse : à 8 mois des élections, la RDC recule de trois places en une année », www.http://popafro.com/liberte-de-la-presse-a-6-mois-des-elections-la-rdc ongo-recule-de-trois-places-en-une-annee/, accédé dernièrement le 22 octobre 2016.

33 Reporters sans frontières, note 32.

34 Marie Soleil FRERE, Elections et médias en Afrique centrale. Voie des urnes, voix de la paix? Paris, 2009 , p27.

35 Arts 36 et 53de la loi n $96-002$ du 22 juin 1996 fixant les modalités d'exercice de la liberté de la presse, Journal officiel, numéro spécial d'Août 2001.

36 Art. 24 de la Constitution du 18 février 2006 telle que modifiée par la loi n ${ }^{\circ} 11 / 002$ du 20 janvier 2011, portant révision de la Constitution de la RDC du 18 février 2006,journal officiel, numéro spécial 5 février 2011. 
Toutefois, s'agissant des médias publics, ceux-ci sont souvent manipulés par le parti au pouvoir afin de transmettre des opinions qui leur sont favorables sous le prétexte de la diffusion d'information publique objective. Concernant l'organisation et le fonctionnement même de la Radio et télévision nationales congolaises, elle est de nature à étouffer toute initiative en province. En effet, elle est sous tutelle du Ministre en charge de la communication qui détient sur elle un pouvoir de contrôle par voie d'autorisation, par voie d'approbation et par voie d'opposition. ${ }^{37}$

Les directives à suivre venant de Kinshasa doivent être respectées à la lettre même si elles ne sont pas de nature à encourager la liberté de la presse. Cette situation s'amplifie en période électorale ou certaines personnes soit de l'opposition ou de la société civile reconnues hostiles au gouvernement se voient refuser l'accès. Leurs activités ne bénéficient pas de la couverture médiatique des médias publics. Le temps de parole alloué aux partis politiques en campagne électorale est disproportionné selon qu'il s'agit d'un parti au pouvoir ou d'un parti de l'opposition ou encore de la société civile. ${ }^{38}$

Lors des scrutins du 28 novembre 2011, le rapport préliminaire de la mission d'observation de l'Union européenne soulignait que « la Radio Télévision Nationale Congolaise (RTNC) n'a pas joué son rôle de service public négligeant le principe d'égalité et d'équilibre en matière d'information. J. Kabila a ainsi reçu $86 \%$ du temps consacré aux candidats présidentiels au journal télévisé, contre 7\% à L. Kengo, 3\% à V. Kamerhe et 1\% à E. Tshisekedi $/{ }^{39}$ Pour mieux garantir la liberté de la presse, il devrait en principe y avoir une certaine interdépendance entre les médias et l'Etat. Ce dernier est appelé à encadrer les médias, à exercer un contrôle sur eux ${ }^{40}$ sans pour autant les soumettre aux pressions politiques. Cela, dans le but de garantir l'égalité d'accès, le pluralisme de l'information, la neutralité de la presse dans le respect de la liberté d'opinion.

En termes de la presse orale et écrite dans la province Sud-Kivu, des plus suivis on retient la radio et la télévision. Pour plusieurs raisons encore (absence de courant électrique, manque de téléviseurs dans certains ménages, la facilité offerte de suivre la radio même à partir d'un téléphone...), la radio est plus suivie que la télévision. ${ }^{41}$

\section{Les Atteintes à la Liberté de la Presse au Sud-Kivu}

Les violations de la liberté de la presse revêtent des formes variées, complexes et parfois aussi déguisées. Il s'agit notamment des enlèvements, tentatives d'assassinat, arrestation,

37 Art 23 du Décret n ${ }^{\circ}$ 09/62 du 03 décembre 2009 fixant les statuts d'un établissement public dénommé Radiotélévision Nationale Congolaise, en sigle « RTNC », $12^{\text {ème }}$ numéro du Journal officiel.

38 Rapport de la mission d'observation des élections de l'Union européenne (MOE-UE), 2011, p.6.

39 Idem.

40 Naupess KIBISWA, « lendemain difficile pour une liberté d'expression fraîchement reconquise ", inparoles d'Afrique centrale: briser les silences, Paris, 2003, p53.

41 La précarité de la vie et l'analphabétisme de la population ne sont pas de nature à les pousser vers cet outil de l'information. 
expulsions, saisies des journaux, menaces, attaque contre les locaux des médias... De ces atteintes, il y en a qui visent directement la personne des agents des médias tels que les assassinats, emprisonnement, enlèvements, menaces, agressions des journalistes... D'autres violations visent les médias eux-mêmes comme la fermeture ou encore la suspension de certaines chaines de radio et de télévision, la coupure du signal, la censure de certaines émissions, les pressions fiscales et financières sur la rédaction... ${ }^{42}$

Le Bureau Conjoint des Nations Unies aux Droits de l'Homme (BCNUDH) a documenté entre le $1^{\mathrm{er}}$ octobre 2011 et le 31 janvier 2012, 42 atteintes à la liberté d'expression et 18 atteintes à la liberté de la presse. ${ }^{43}$ Et même après les jours des scrutins, les atteintes à la liberté de la presse se sont faites plus nombreuses afin d'empêcher toute manifestation de mécontentement quant au déroulement du processus électoral et toute contestation des résultats.

L'ONG Journaliste en danger (JED) a dit avoir rapporté 52 cas de violations des droits d'informer et d'être informé en RDC entre janvier et mai 2013, soit une moyenne de trois cas d'atteinte à la liberté de la presse par semaine. ${ }^{44}$ Pour ceux qui plaident pour la baisse des cas d'atteinte à la liberté de la presse en RDC depuis lors, cette ONG affirme qu'elle serait les fruits de l'autocensure et de la censure auxquelles les journalistes sont confrontés au quotidien. ${ }^{45}$ Et pour l'année 2015, 72 atteintes à la liberté de la presse ont été répertoriées par cette même organisation dont deux assassinats de journalistes, ${ }^{46} 22$ autres privés de liberté pendant des périodes plus au moins longues, 25 journalistes agressés ou menacés dans l'exercice de leur profession et c'est sur l'ensemble du territoire national. ${ }^{47}$

Le Sud-Kivu n'enest pas épargné puisque de ces 72 cas inventoriés dans toute la République, cette province à elle seule est concernée par 12 cas. ${ }^{48}$ Dans une telle situation, la tendance consiste pour certains médias à demander la protection de certains hommes politiques avec comme conséquence, qu'au moment de la campagne, leur couverture offre plus d'avantages à ceux qui les ont parrainés. Nombreuses violations de la liberté de la presse ont été inventoriées au Sud-Kivu par différents acteurs intervenants dans ce domaine. D'où, il y a lieu de clarifier certaines d'entre elles qui méritent davantage de détails.

42 FRERE, note 34,p.27.

43 Bureau Conjoint des Nations Unies aux Droits de l'Homme, Rapport sur les Atteintes à la Liberté d'Expression et à la Liberté de la Presse du $1^{\mathrm{er}}$ octobre 2011 au 31 janvier 2012.

44 Rapport annuel 2015, Journaliste en danger,RDC : atteintes à la liberté de la presse, www.7sur7.cd consulté le 15 juillet 2016.

45 Rapport annuel 2015, Journaliste en danger,RDC, note 44.

46 Il s'agit de Mr Robert Chimwami Shalubuto de RTC/Goma et Soleil Balanga de la Radio communautaire de Mokoto.

47 Journaliste en danger (JED), Rapport annuel RDC 2012, www.afrikarabia.com/l'Etatde la liberté de la presse en Afrique centrale francophone, accédé dernièrementle 26 août 2016.

48 Le Potentiel, « interdiction de diffusion du film « l'homme qui répare les femmes: la colère d'Hypocrate ». Dr Mukwege dénonce le refus du droit d'accès à l'information », disponible sur www.lepotentielonline.com, consulté le 26/10/2016. 
a. Les assassinats et agressions des journalistes

La seule ville de Bukavu a connu entre 2006 et 2016, trois assassinats des journalistes dont Serge Maheshe Kasole (2007), Didace Namujimbo (2008) et Koko Bruno Chrirhambiza (2010). ${ }^{49}$ Pour le dernier cité, jusqu'à ce jour l'issu des enquêtes reste inconnu, aucun suspect n'a été appréhendé.

Pour 2016, le climat politique étant devenu tendu par le fait que les élections ne sont pas organisées dans le délai constitutionnel,des cas d'insécurité sont couramment signalés dans tous les coins de la province. Ce qui rend la tâche compliquée aux journalistes empêchés de faire entendre l'autre son de cloche. ${ }^{50}$

\section{b. Emprisonnement}

La RDC affiche un tableau sombre avec quatre journalistes derrière les barreaux, cinq chaines de télévision proches de l'opposition fermées depuis la fin des élections de novembre 2011, un journal suspendu depuis plus d'une année, deux films-documentaires réalisés par des journalistes censurés et interdits de diffusion... ${ }^{51} \mathrm{~S}$ 'agissant des arrestations arbitraires, l'Agence Nationale des Renseignements (ANR) a été plusieurs fois citée par des associations des médias et des organisations de défense des droits humains pour avoir agressé violemment les journalistes et de les avoir détenu arbitrairement dans ses installations. L'organisation journaliste en danger avait dénoncé le 10 mai 2016, l'arrestation des journalistes au Sud-Kivu accusant aussi les forces de l'ordre d'avoir attaqué des stations de radio émettant dans cette région. ${ }^{52}$ En marge de la journée internationale de la liberté de la presse célébrée le 03 mai de chaque année, cette organisation avait recensé 15 cas de journalistes emprisonnés. ${ }^{53}$

\section{c. Menaces et intimidations}

Les menaces et intimidations contre les journalistes sont monnaies courantes au Sud-Kivu à l'approche des élections. ${ }^{54} \mathrm{~A}$ titre illustratif, la dégradation de la liberté de la presse à l'approche de l'année 2016, prévue pour la fin de mandat du Président de la République en

49 Centre Olame, « la journée internationale de la presse au Sud-Kivu », disponible sur http://www.ol ame.org/2012/05/ accédé dernièrement le 24 Août 2016.

50 Journaliste en danger (JED), Rapport annuel RDC 2012, note 47.

51 Le Potentiel, « interdiction de diffusion du film « l'homme qui répare les femmes : la colère d'Hypocrate ». Dr Mukwege dénonce le refus du droit d'accès à l'information », disponible sur www.lepotentielonline.com, accédé dernièrementle 26/10/2016.

52 Radio Okapi, « JED dénonce les arrestations des journalistes au Sud-Kivu »,disponible sur www.radiookapi.net accédé dernièrement le19 Août 2016.

53 Radio Okapi, Jed dénonce les arrestations des journalistes au Sud-Kivu, disponible sur www.radiookapi.net/, accédé dernièrementle 26 aout 2016.

54 Reporteurs sans frontières, note 3, p. 15. 
RDC au regard de l'article 70 de la Constitution. ${ }^{55}$ Dans ce contexte, la presse est particulièrement malmenée. Le pouvoir harcèle de plus en plus les organes de presse. Les détentions arbitraires, les coupures des signaux et les intimidations sont tellement fréquentes et à force de répétition deviennent une habitude. Nombreux journalistes sont menacés et agressés par de hauts responsables civils ou militaires pour leur empêcher de donner de l'espace à la société civile ou encore à l'opposition critiques vis-à-vis du pouvoir en place. ${ }^{56}$

\section{d. Fermeture et suspension des radios et télévisions}

Selon Reporteurs Sans Frontières (RSF), une dizaine de médias ont été soit fermés, soit suspendus, des émissions interdites, du matériel confisqué et des locaux saccagés. ${ }^{57}$ Plusieurs chaînes de radio et detélévisions, à l'exemple de la radio France internationale (RFI), ont vu leur signal coupé par les autorités. La plupart de violations de la liberté de la presse relèvent en particulier de l'abus de pouvoir de la part des autorités nationales ou même provinciales. Alors que la décision est censée être prise au niveau du Conseil Supérieur de l'Audiovisuel Congolais (CSAC), certains médias se sont vus fermés ou suspendus de fonctionnement par les autorités sans toute forme de procès. ${ }^{58}$ Parfois, devant pareille situation, le CSAC prenait une décision confirmant la décision déjà prise pour régulariser la situation, et cela en violation des lois congolaises. ${ }^{59}$ On peut noter également l'impunité dont jouissent les auteurs des violations de la liberté de la presse favorisant la recrudescence de ces cas puisque les auteurs se savent hors de portée. ${ }^{60}$

La question de la liberté de la presse et des entraves à celle-ci n'est pas à analyser du seul point du vue des abus les autorités en place sont souvent auteurs. Elle est vue aussi du point de vue des manquements dans le chef des certains médias, qui en abusent. Des abus peuvent provenir du non respect par eux de certaines règles d'éthique et déontologie du journaliste congolais dont ils doivent faire preuve en tout temps y compris en période électorale. Le constat est que certains journaux et stations de radio et de télévision jouent un

55 Art 70 de la Constitution du 18 février 2006 «Le Président de la République est élu au suffrage universel direct pour un mandat de cinq ans renouvelable une seule fois ». Ayant été élu en 2006 et renouvelé son mandant en 2011, en principe, le Président congolaisne peut plus se porter candidat aux prochaines élections.

56 Reporteurs sans frontières, note 32, p. 17.

57 Reporteurs sans frontières, note 32, p. 17.

58 Ibidem

59 Rapport du BCNUDH, Pour un recensement exhaustif des mesures de coupure de radios et de télévisions entre les 1eroctobre et 31 décembre 2011 sur le territoire de la RDC, qu'elles soient légales ou non, p.55.

60 Malgré la recrudescence des violations de la liberté de la presse, aucune action judiciaire n'a été ouverte contre une personnalité quelconque pour qu'elle en réponde. 
rôle de «presse de propagande, déterminée à défendre les intérêts politiques de leurs propres candidats et à diaboliser leurs adversaires politiques $\gg .{ }^{61}$

Dans une province où de nombreux organes de presse privés sont détenus par des candidats et des partis politiques, ces violations peuvent déboucher sur la violence, l'incitation à la à la vengeance voire sur l'apologie du crime. Comportements que tout journaliste devrait à tout prix éviter. Parfois, même les médias de l'Etat participent à envenimer la situation des violations de la liberté de la presseen se mettant au service quasi exclusif du parti au pouvoir. ${ }^{62}$ Devant un tel tableau des violations de la liberté de la presse, interrogeonsnous sur le rôle des médias pendant la période électorale. ${ }^{63}$ Autrement-dit, qu'attendre de ceux-ci durant cette période cruciale de la vie politique d'un peuple?

\section{B. ROLE DES MEDIAS PENDANTLE PROCESSUS ELECTORAL}

De manière générale, la mission principale des médias est d'informer, de former et de divertir la masse. Ils exercent cette mission en observant le milieu environnant, en assurant la communication sociale, en fournissant une image au monde, en transmettant la culture, en contribuant au bonheur des autres et enfin en servant d'espace pour la publicité. ${ }^{64}$ Les périodes pré-électorale, électorale et postélectorale sont des moments privilégiés d'information publique. A ce titre elle joue un rôle de surveillance, garantit la transparence du processus et assurela fiabilité des sondages. ${ }^{65}$

Le principe directeur qui sous-tend le rôle des médias dans les élections est que « sans liberté de presse et le pluralisme médiatique, la démocratie ne peut exister $\gg{ }^{66}$ Les médias jouent donc un rôle indispensable dans la démocratisation et la construction d'un Etat de droit. A cet effet, ils sont qualifiés de quatrième pouvoir par certains. ${ }^{67}$ Cette qualification est faite par allusion aux trois pouvoirs constitutionnels (législatif, exécutif et judiciaire) dans le processus de la formation de l'opinion publique. C'est aussi en raison de l'influence que la révélation de certains faits peut avoir dans les prises de position des citoyens au moment d'opérer leur choix. Les faits et analyses qu'ils rapportent, sont souvent déterminants, en période électorale pour préserver la transparence du processus. Ce rôle est habituelle-

61 JurisPedia, « liberté de la presse en période électorale » disponible sur www.cairn.info, consulté le 10 octobre 2016.

62 FLESHMAN, note 15, p.9.

63 Entendue ici la période préélectorale, celle électorale et même post électorale.

64 Jean Claude BERTRAND, La déontologie des médias, Paris, 1997 p. 15.

65 F. BALLE, Medias et société, presse audiovisuelle, télécommunication et internet, 11è éd. Paris, 2003, p 657.

66 Marie Soleil FRERE, Pas d'élections démocratiques sans médias libres et pluralistes, " Afrique contemporaine » avril 2011, p. 48.

67 Association pour une constituante, « De qui le quatrième pouvoir est-il le nom »disponible sur www.pouruneconstituante.fr, accedé dernièrement le 18 octobre 2016. 
ment vu comme celui d'un « surveillant ». ${ }^{68} \mathrm{La}$ transparence telle qu'exigée pour l'accès à l'information signifie que l'électorat dispose des informations nécessaires et suffisantes pour faire un choix rationnel. ${ }^{69}$

Le rôle que les médias sont appelés à jouer diffère selon qu'il s'agit de la période préélectorale, électorale ou encore postélectorale.

\section{Le rôle des médias dans la période préélectorale}

Durant cette période, les médias peuvent jouer un rôle plus spécifique en facilitant la pleine participation de tous aux élections. Dans ce cas, ils servent d'outils dont peuvent se servir les parties prenantes pour appeler les électeurs à se rendre aux urnes. Ils participent à l'analyse des performances du gouvernement. Le fait pour eux de poser un regard critique sur les échecs et les réussites du gouvernement congolais, les amène à informer le public sur l'efficacité de ses représentants et l'aider à exiger des comptes. ${ }^{70}$

Les médias ont un mandat d'informer les citoyens sur les partis politiques et les candidats participant aux élections et leurs programmes. De cette manière, ils contribuent à la formation de l'opinion de l'électorat et à l'éducation des électeurs sur la manière d'exercer leurs votes.Les médias peuvent ainsi être un outil non seulement pour informer les électeurs sur les enjeux et les candidats, mais aussi pour véhiculer des renseignements de base sur la façon de voter ou sur l'objet du vote. « La presse, en coordination avec les autres instances de régulation et d'autorégulation constituent une voie incontournable d'éducation, de formation (de l'opinion) et d'information de la population. $\rangle^{71}$

Par la couverture de la campagne électorale, ils offrent aux partis politiques un moyen de communiquer avec leur électorat. Ne pouvant être en contact direct avec leurs électeurs, les candidats et leurs partis passent par les médias. S'il faut se déplacer pour suivre le discours de tel ou tel autre, ne peuvent s'y rendre que ceux qui y trouvent un intérêt particulier. Alors que, grâce à la campagne faite dans les médias, les candidats ont encore la chance de convaincre les indécis qui peuvent être séduits par leurs projets de société. Cela peut aller jusqu'à amener certains électeurs à revoir leur choix en disposant des éléments sur les programmes des candidats dont ils auraient suivi les déclarations même via la presse. Ceci s'expérimente d'avantage à travers la culture des débats radiodiffusés et/ou télévisés entre différents protagonistes. Garantir à tous les partis politiques et aux candidats l'accès égal aux médias est donc primordial à la démocratie. Les candidats et les partis ont un droit ex-

68 Exposé de $M$. NKUNDIYE au séminaire du SPAD sur la liberté de la presse et couverture médiatique des élections, Août 2016.

69 FRERE, note 66, p. 49.

70 KAYEMBE, note22,p. 30.

71 D. NKINGI, Le rôle des medias dans la réconciliation et dans la reconstruction de la République démocratique du Congo», rapport pour le Séminaire International sur la gestion de la transition en République Démocratique du Congo, p. 245. 
plicite de fournir des informations aux électeurs concernant leurs compétences, leurs programmes politiques et les projets qu'ils proposent. ${ }^{72}$

Les campagnes des candidats et des partis sont bien sûr une forme de débat, mais d'autres voix devraient également se faire entendre dans les forums publics comme le dispose le Pacte international relatif aux droits civils et politiques, « tous les peuples ont le droit d'exprimer des idées et opinions contraires. ${ }^{73}$ En général, la participation des médias à la mise en place de cet espace de débat et de discussion est vitale. Les médias fournissent aux citoyens ordinaires un mécanisme leur permettant de se faire entendre. Une manière pour eux d'essayer d'influer sur les agendas et programmes politiques des candidats en période de campagne, et parfois d'obtenir le soutien d'autres électeurs et de les influencer. ${ }^{74}$

Par la couverture médiatique des campagnes, les électeurs ont la chance d'avoir des informations impartiales à travers des mesures telles que la répartition équitable du temps d'antenne entre tous les partis et candidats participant aux élections, des accords concernant des programmes d'information vérifiées des rapports et des émissions ou des débats entre les dirigeants des partis. Il est donc essentiel de s'assurer que tout parti politique ou candidat indépendant ait accès aux médias, notamment la radio et/ou la télévision.

Le principe d'impartialité et de neutralité imposé au journaliste lui oblige de s'abstenir de toute intervention en faveur ou contre un candidat ou une formation politique car «s'il appartient aux stations de radiodiffusion et la télévision d'informer les auditeurs sur le déroulement de la campagne, elles ne sauraient diffuser surtout les éléments de polémique au profit ou au détriment d'un candidat. ${ }^{75}$ " La loyauté et la dignité veulent qu'ils s'abstiennent des fausses déclarations de soutien, fausses déclarations du candidat, tract anonymes. $»^{76}$

En 2006 et 2011, du fait des grandes distances et du coût élevé des déplacements ainsi que de la présence continuelle de milices armées partisanes dans certains coins de la province duSud-Kivu, les campagnes électorales menées habituellement par des candidats et des représentants de partis étaient restreintes. ${ }^{77}$ Dans ces conditions, la responsabilité des médias était alourdie dans la mesure où ils constituent le principal moyen d'informer les électeurs et de faire campagne.

72 KHNEL, note 26, p. 73.

73 Pacte international relatif aux droits civils et politiques, Haut-commissariat des Nations Unies aux droits de l'homme, consulté le 16 août 2012, http://www2.ohchr.org/french/law/ccpr.htm accédé dernièrementle 12 Août 2016.

74 HOWARD, Les médias et les élections, IMPACS, 2005, p. 8, disponible sur www.portal.unesco.org accédé dernièrementle 25 Août 2016.

75 HOWARD, note 74, p. 14.

76 Idem.

77 B. ROZUMILOWICZ, « Democratic change : atheoretical perspective », in Media Reform, Democratizing the media, democratizing the state, éd. préparée par Price E., Monroe, Beata Rozumilowicz, Stephan G.Verhulst. London: Routeledge, p. 26. 
Le rôle des médias ne se limite pas à la seule période des campagnes. Ils ont d'autres responsabilités y compris pendant les élections proprement-dites.

\section{Le rôle des médias pendant dans les élections}

Les médias sont essentiels à la démocratie. ${ }^{78}$ Durant la période électorale, les médias indépendants et pluralistes sont indispensables pour garantir la transparence, la responsabilité et la participation, aspects fondamentaux de la bonne gouvernance et du développement fondé sur les droits de l'homme. La question reste entière pour les médias qui seraient manipulés soit par le pouvoir soit par les particuliers à travers les répressions, ou par corruption étant donné que la plupart d'entre eux appartiennent aux personnalités politiques.

Le rôle des médias ne s'arrête pas à ce que peuvent être leur prestation en période de campagne. Pendant la tenue des scrutins, ils observent la manière dont le vote est fait, surveillent le dépouillement et rapportent le moindre incident s'il y en a eu qui aurait survenu pendant le vote. Lors de la publication des résultats, son rôle est accru parce qu'alors, la population après s'être exprimé éprouve un grand besoin de connaître l'issu de leur vote. Et pour atteindre la majorité de la population, la CENI fait recours à la presse pour diffuser les résultats des élections. ${ }^{79}$

Les médias sont donc les principaux témoins du déroulement des scrutins électoraux. Ils jouent le rôle de veille le jour du scrutin et contribuent à garantir la transparence des opérations de vote et de dépouillement. Les journalistes, pour leur part, sont observateurs, moniteurs et régulateurs du processus électoral. ${ }^{80}$

\section{Le rôle des médias dans la période postélectorale}

Les médias jouent aussi un grand rôle même après les élections en suivant de près le processus électoral lui-même dans le but d'auditer et d'évaluer son équité, son efficacité et son intégrité. Ils jouent donc un rôle important durant la période qui suit la clôture du scrutin et l'annonce des résultats officiels. Cette période est le moment où les voix de la contestation s'élèvent. Les médias sont une source officielle de l'information pour les résultats. Ainsi, pour éviter que les rumeurs se répandent dans un vide d'information la pratique est de concevoir un processus qui publie les résultats provisoires dès qu'ils sont disponibles. ${ }^{81}$

A cette occasion, ils offrent à nouveau de l'espace aux différentes parties prenantes pour s'exprimer sur la manière dont les élections se sont passées. Dans cette optique, ils peuvent être un moyen de contester ou de critiquer la manière dont les scrutins se sont déroulés.

78 A-M GINGRAS, Médias et démocratie. Le grand malentendu, Québec, 2001, p. 200.

79 Reporters sans frontières, note 3, p. 10.

80 Exposé de M. NKUNDIYE, note 68, p.17.

81 Programme des Nations unies pour le développement (PNUD), Médias et élections, un guide pour les praticiens électoraux, p. 16. 
Ce rôle des médias comme nous venons de le voir, crée dans le chef du citoyen, un devoir moral d'être informé, qui fait partie de sa responsabilité à l'égard de la communauté politique ${ }^{82}$.

Bref, les médias affectent sans doute la politique en apportant aux électeurs des informations pertinentes, car mieux informés, ils votent pour des responsables politiques qui défendent leurs intérêts. Ils influencent aussi la façon dont les hommes politiques réagissent à l'évolution et à la demande de prestations publiques. ${ }^{83}$ De ce fait, il est important que les électeurs soient bien informés.

On assiste cependant à des multiples formes de manipulation de la presse durant le processus électoral. Ceci peut partir de l'omission consistant à supprimer certains faits significatifs ou peu favorable à la thèse défendue, à la couverture des détails susceptibles de susciter une opposition. Il peut s'agir aussi du retardement d'un message jusqu'au moment où le destinataire n'aura plus le temps de réagir ou encore le fait de rendre le message plus difficile à percevoir par le destinataire. C'est aussi le cas de l'enrôlement des faits exacts dans un flot de rumeurs nébuleuses pour créer la confusion dans le chef du destinataire... ${ }^{84}$

\section{CONCLUSION}

Toute personne a droit à l'information. La liberté de la presse, d'information et d'émission par la radiodiffusion sonore et la télévision, la presse écrite ou tout autre moyen de communication des masses sont garanties sous réserve du respect de l'ordre public, de bonne mœurs et des droits d'autrui. ${ }^{85}$ " Aucun journaliste ou professionnel des médias, ne peut être inquiété ou de quelque manière que ce soit dans l'exercice régulier de sa profession, ni se voir interdire l'accès aux sources d'information $»{ }^{86} \mathrm{Tel}$ est le principe. Les médias sont appelés à jouer leur rôle comme il se doit, celui de gardien et surveillant de la transparence et de la bonne conduite des affaires publiques, informateur du public, éducateur de la population et facilitateur de l'échange entre les décideurs et la population.

Il résulte cependant du tableau peint dans les lignes précédentes à la lumière de la pratique qui est faite de la liberté de la presse, particulièrement en période électorale, que ce

82 D. ELLIOT(dir) Responsible journalist, Beverly, Hills, New bury Park, London, New Dehli, sage 2è éd, 1998 p. 7.

83 Institut Panos Paris, Régulation des médias dans les Grands lacs : défendre la liberté de la presse ou discipliner les acteurs des médias? Paris, novembre 2005.

$84 J$. BANGA, Journaliste : à la croisée des chemins! Déontologie, loi et éthique sur le parcours journalistique, Kinshasa,Médias Paul, 2006, p.35.

85 M. KALAMBAYI, Module de formation : droit,libertés publiques et devoirs : exercice des mécanismes de promotion et protection en RDC. Cas de la presse, disponible sur www.cefopdh.org/ dernièrement accédéle 23 Août 2016.

86 Art. 5 Loi organique $\mathrm{n}^{\circ} 11 / 001$ du 10 janvier 2011 portant composition, attribution et fonctionnement du Conseil Supérieur de l'Audiovisuel et de la Communication, www.leganet.cd/Legislation/ JO/2011/JOSdernièrement accédé le 18 juillet 2016. 
principe demeure au Sud-Kivu, un mythe, un idéal vers lequel tout Etat de droit devait aboutir.

Il résulte des analyses faites précédemment que la situation de la liberté de la presse dans le contexte électoral au Sud-Kivu est alarmante. Cela s'explique par nombreux obstacles auxquels les professionnels des médias et les maisons de presse sont régulièrement confrontés dans pareille période. Le rôle primordial que joue la presse pendant ce temps-là, conduit même à son étouffement. Instrument essentiel de communication entre les électeurs et les candidats voire les partis politiques, la presse subit d'énormes pressions qui vont jusqu'à entraver sa liberté. Elle est souvent confrontée à des nombreux obstacles liés à plusieurs facteurs. Parmi eux on retient, la précarité de leurs conditions de travail et le déficit d'encadrement des journalistes. Le mode de financement des médias a une répercussion sur le contenu des messages qu'ils portent à la connaissance des auditeurs et sur l'exercice de la liberté de la presse. Au Sud-Kivu, au sein des médias privés, nombreux n'ont pas de contrat de travail avec les médias pour qui ils travaillent. Leur rémunération relève du bon vouloir des propriétaires ${ }^{87}$.

Au Sud-Kivu, il n'existe pas une seule université qui organise en son sein une faculté de journalisme. Sauf pour quelques uns et qui sont rares d'ailleurs à avoir suivi une formation ailleurs, la majorité se forme par l'expérience pendant qu'ils exercent déjà comme journalistes. Les modalités d'accès au métier des journalistes devraient être encadré car un journaliste bien formé exerce son métier de façon professionnelle et s'adapte facilement aux mutations constantes de la profession. Il faudra pour cela, inclure le programme académique de journalisme pour s'assurer de la formation adéquate des médias. Sans pour autant que cela pèse sur la liberté de la presse, l'Etat pourrait subventionner les médias pour endiguer dans une certaine mesure, le problème des moyens financiers auxquels ils sont couramment confrontés.

Le contrôle de certains médias par des personnalités politiques et des opérateurs économiques contribue largement aux violations de la liberté de la presse. Pour espérer une presse un peu plus libre y compris en période électorale, cela implique que l'Etat favorise l'accès aux médias de services publics. Ce qui suppose aussi qu'il garantisse l'indépendance des instances de régulation des médias, et facilite l'exercice de la liberté syndicale de ceux-ci. Il faudrait également que les médias s'opposent à toute répression du pouvoir, à toute corruption de manière qu'ils soient règlementés par un régulateur indépendant afin qu'ils arrivent à produire une information exacte, objective, pouvant aider les électeurs dans toute étape des élections à faire un choix éclairé.

Les atteintes à la liberté de la presse étant aussi œuvre des journalistes eux-mêmes, une vulgarisation du Code d'éthique et déontologie du journaliste congolais est essentielle également pour éviter les dérives professionnelles.

87 Entretien avec le président de l'union congolaise de presse, antenne de Bukavu, le 23 décembre 2015. 
La presse ne peut jouer pleinement son droit de « publier, communiquer à autrui, diffuser librement des idées, informations et connaissances sur les droits de l'homme et les libertés fondamentales $»^{88}$ en période électorale que si sa liberté est garantie et pratiquée. Et comme il n'y a pas de liberté sans responsabilité, les professionnels des médias devraient se conformer aux règles en la matière. L'Etat quant à lui devrait donc assainir l'espace médiatique en assurant le respect aux personnes investies de la mission d'informer, en sanctionnant les auteurs d'atteinte à la liberté de la presse. L'exercice de la liberté de la presse étant étroitement lié aux luttes politiques particulièrement en période électorale, sa reconnaissance constitutionnelle lui assigne une certaine primauté. Cela s'explique par le fait que les autres textes à valeur inférieure doivent s'y conformer.

\section{REFERENCES}

\section{Textes juridiques}

Constitution du 18 février 2006 telle que modifiée par la loi n ${ }^{\circ} 11 / 002$ du 20 janvier 2011, portant révision de la Constitution de la RDC du 18 février 2006, in journal officiel, numéro spécial 5février 2011

Pacte international relatif aux droits civils et politiques, Haut-commissariat des Nations Unies aux droits de l'homme, http://www.ohchr.org/french/law/ccpr.htm consulté le 16 août 2012

Déclaration Universelle des Droits de l'Homme du 10 décembre 1948

loi nº6-002 du 22 juin 1996 fixant les modalités d'exercice de la liberté de la presse en

Loi organique $\mathrm{n}^{\circ} 11 / 001 \mathrm{du} 10$ janvier 2011 portant composition, attribution et fonctionnement du Conseil Supérieur de l'Audiovisuel et de la Communication

Code d'éthique et de déontologie du journaliste congolais, in journal officiel, numéro spécial du 20 juin 2006

Décret n09/62 du 03 décembre 2009 fixant les statuts d'un établissement public dénommé Radiotélévision Nationale Congolaise, en sigle « RTNC»

Edit provincial portant protection des défenseurs des droits humains et des journalistes dans la province du Sud-Kivu de février 2006

\section{Doctrine}

1. Association des journalistes professionnels (APJ), les journalistes et leurs sources. Guide de bonnes pratiques, mars 2012.

2. BANGA J., Journaliste : à la croisée des chemins! Déontologie, loi et éthique sur le parcours journalistique, Médias Paul, Kinshasa, 2006.

88 Art 5 point c) de l'Edit provincial portant protection des défenseurs des droits humains et des journalistes dans la province du Sud-Kivu de février 2006 disponible sur www.protectioninternational. org/fr/2016/02/21/rdc-un-nouveau-cadre-juridique-au-sud-kivu-pour-la-protection-des-defenseursdes-droits-de-lhomme-2 dernièrement consulté le 23 novembre 2016. 
3. BCNUDH, Pour un recensement exhaustif des mesures de coupure de radios et de télévisions entre les 1eroctobre et 31 décembre 2011 sur le territoire de la RDC.

4. BERTRAND Jean-Claude, La déontologie des médias, Paris, PUF « que sais-je? », 1997.

5. CORNU Daniel., Journalisme et vérité pour une éthique de l'information, Genève, éd. Labor, 1994.

6. DARRACQ Vincent et Victor MAGNANI, «Jeux de pouvoir en Afrique : le Nigéria et l'Afrique du Sud face à la crise ivoirienne ", Politique étrangère, Vol 76, n², juin 2006.

7. ELLIOT D.(dir) Responsible journalist, Beverly, Hills, New bury Park, London, New Dehli, sage 2è ed, 1998.

8. Francis BALLE, Medias et société, presse audiovisuelle, télécommunication et internet,., Paris, 11è éd Montchrestien, 2003.

9. FLESHMAN Michael, un.org/africarenewal/fr/magazine/april-2007/rdc-les-médiasdéfendent-la-démocratie, avril 2007.

10. GUENARD François., "La promotion de la démocratie : une impasse théorique?», Esprit $\mathrm{n}^{\circ} 341$, janvier 2008.

11. GUILLEBAUD Jean-Claude., Les médias contre la démocratie? Ed. Esprit www.jstor.org/stable/24276508Accessed: 23-08-2016 12:08.

12. HOWARD R., les médias et les élections, IMPACS, 2005, www.unesco.org consulté le 25 août 2016.

13. Institut Panos Paris, Régulation des médias dans les Grands lacs : défendre la liberté de la presse ou discipliner les acteurs des médias? éd. Panos Paris, novembre 2005.

14. KABEYA M, Regard sur la presse du Congo-Belge à celui de Kabila sans oublier le Zaïre de Mobutu, Paris, L'Harmattan, 2003.

15. KAYEMBE Aimé. et alii, Situation des médias en RDC, Paris, éd Institut Panos paris, 2004

16. KIBISWA Naupess., " lendemain difficile pour une liberté d'expression fraîchement reconquise », in paroles d'Afrique centrale : briser les silences, éd. Karthala, Paris, 2003.

17. KHNEL J., Enjeux de la bonne gouvernance et rôle des médias en RDC, in Médias et bonne gouvernance, Kinshasa, 2005.

18. MB A-M GINGRAS, Médias et démocratie. Le grand malentendu, Québec, Presses de l'Université duQuébec, 2001.

19. Marie-Soleil FRERE «Pas d'élections démocratiques sans médias libres et pluralistes », Afrique contemporaine, avril 2011, $\mathrm{n}^{\circ} 240$.

20. Marie-Soleil FRERE, Elections et médias en Afrique centrale. Voie des urnes, voix de la paix? Paris, Karthala, 2009.

21. NKINGI D., Le rôle des medias dans la réconciliation et dans la reconstruction de la République démocratique du Congo», rapport pour le Séminaire International sur la gestion de la transition en République Démocratique du Congo. 
22. PNUD, Médias et élections, Guide pour les praticiens électoraux

23. Reporters sans frontières, Guide pratique du journaliste en période électorale, RSF et OIF, 2015.

24. ROZUMILOWICZ B., "Democratic change: atheoretical perspective », in Media Reform, Democratizingthe media, democratizing the state, éd. préparéepar Price E., Monroe, Beata Rozumilowicz, Stephan G.Verhulst. London : Routeledge. 


\section{Impressum}

\section{KAS African Law Study Library - Librairie Africaine d'Etudes Juridiques}

Herausgeber: Konrad-Adenauer-Stiftung e.V., Klingelhöferstraße 23, 10785 Berlin

Schriftleitung: RA Prof. Dr. Hartmut Hamann, Falkertstraße 82, D-70193 Stuttgart Telefon: +49 (o) 711 120950-30 | Fax : +49 (o) 711 120950-50

Mail: hamann@hamann-legal.de

Erscheinungsweise: 4 Ausgaben pro Jahr

Druck und Verlag: Nomos Verlagsgesellschaft mbH \& Co. KG - Waldseestr. 3-5 D-76530 Baden-Baden. Telefon +49-7221-2104-0 / Fax 49-7221-2104-27 E-Mail:nomos@nomos.de

Anzeigen: sales friendly Verlagsdienstleistungen - Inh. Frau Bettina Roos - Pfaffenweg 15 - D-53227 Bonn Telefon +49-228-97898-o / Fax +49-228-97898-20 E-Mail:roos@sales-friendly.de

Urheber- und Verlagsrechte: Die Zeitschrift und alle in ihr enthaltenen einzelnen Beiträge sind urheberrechtlich geschützt. Jede Verwertung außerhalb der engen Grenzen des Urheberrechtsgesetzes ist ohne Zustimmung des Verlages unzulässig. Das gilt insbesondere für Vervielfältigungen, Übersetzungen, Mikroverfilmungen und die Einspeicherung und Verarbeitung in elektronischen Systemen. Der Nomos Verlag beachtet die Regeln des Börsenvereins des Deutschen Buchhandels e.V. zur Verwendung von Buchrezensionen.

ISSN 2363-6262

\section{Nomos}

